\title{
Centrosome Functions as a Molecular Dynamo in the Living Cell
}

\author{
Yue Zhao \\ Department of Radiation Oncology, University of Texas Southwestern Medical Center, Dallas, USA \\ Email: alexanderyz@gmail.com
}

Received 16 June 2015; accepted 17 July 2015; published 20 July 2015

Copyright (C) 2015 by author and Scientific Research Publishing Inc. This work is licensed under the Creative Commons Attribution International License (CC BY). http://creativecommons.org/licenses/by/4.0/ c) (i) Open Access

\begin{abstract}
Recent development in the field of quantum biology highlights that the intracellular electromagnetic field (EMF) of microtubules plays an important role in many fundamental cellular processes such as mitosis. Here I propose an intriguing hypothesis that centrosome functions as molecular dynamo to generate electric flow over the microtubules, leading to the electric excitation of microtubule EMF that is required for spindle body microtubule self-assembly. With the help of motors proteins within the centrosome, centrosome transforms the energy from ATP into intracellular EMF in the living cell that shapes the functions of microtubules. There will be a general impact for the cell biology field to understand the mechanistic function of centrosome for the first time in correlation with its structural features. This hypothesis can be tested with technics such as super resolution live cell microscope.
\end{abstract}

\section{Keywords}

Centrosome, Dynamo, Electro Magnetic, Microtubule, Electron

\section{Introduction}

Centrosome was first discovered by Theodor Boveri in the 1880's [1], it is the key organelle that is responsible for mitosis and meiosis in metazoan lineage of eukaryotic cells [2]. In animal cells, centrosome regulates the nucleation and spatial organization of microtubules, functioning as the primary microtubule-organizing center (MTOC) [3]. The centrosome is comprised of two centrioles that are surrounded by pericentriolar material (PCM). The two centrioles are perpendicularly arranged, one centriole has additional appendages at the end farthest from the other centriole (distal) and is called the mother or maternal centriole, the subdistal appendages of 
the maternal centriole also act like microtubule-anchoring sites [4] [5]. Pelletier et al. reported that subdiffraction imaging of centrosomes revealed pericentriolar material which had higher-order organizational features. Centrosome components adopt a toroidal pattern with progressively larger, overlapping diameters around the proximal end of the mother centriole in interphase cells. On one side, the toroid is slightly opened (gap) in the area where the daughter centriole is positioned [6], this higher order structural feature of centrosome may help the mother and daughter centriole to form an orthogonal configuration. In most of cases, each centriole is composed with 9 MT triplets and is $\sim 0.5 \mu \mathrm{m}$ in length and $0.2 \mu \mathrm{m}$ in diameter [7] [8].

Recent studies in the field of quantum biology point to the possibility that electric magnetic interactions may involve in many fundamental cellular processes [9] [10]. In particular, the electromagnetic property of microtubule has been reported with both computation modelling and experimental evidences, Cifra et al. used computation model to simulate the electric pulse moving along microtubules, Bandyopadhyay et al. reported that nano sized electric pump was required for the self-assembly of microtubules in live cell simulator[11]-[13]. Medical treatment of cancer with cancer cell specific interfering EMF has been developed to disrupt the mitotic spindle microtubules of cancer cells [14]. Centriole produces an electromagnetic field apparently due to the longitudinal oscillation of its microtubules (MTs). Centrosome clustering is a hallmark of cancer cells. A cluster of centrioles is therefore presumed to produce an enhanced electromagnetic field. It is possible to target cancer cells using nano particles based on the enhanced electromagnetic property of cancer cells [15]. However, some important questions remain unanswered, what is the energy source of the intracellular electric field and what is the molecular mechanism that leads to the excitation of intracellular electric field? ATP is the most common cellular energy source. To transform the chemical energy in ATP into electric magnetic field within the living cell, cell needs to have a molecular dynamo to transform the mechanistic movement of protein complexes to directional movements of intracellular electrons, leading to the electric excitation of the spindle body microtubules as well as the $\mathrm{M}$ phase chromosomes, which is essential for mitosis [9].

\section{Hypothesis}

Here I present a novel hypothesis that centrosome functions as a molecular dynamo in the living cell to generate electric current from the cytosol electrolyte to the spindle body and $\mathrm{M}$ phase chromosome, leads to the electric excitation of the spindle body and chromosome during mitosis. Based upon the structure of the centrosome, there is one microtubule in the center, and 9 microtubule triplicate outside, connected by motor proteins such as dynein and kinesin [3] (https://vimeo.com/58347006). The mechanistic movement of these motor proteins will trigger the rotation of the microtubules triplets forming the barrel structure of the centriole to rotate around the center microtubule. The rotation and electric oscillation of each centriole will generate a dynamic electromagnetic field that mimic the physical structure of the centriole, and the orthogonal arrangement of centrioles of each centrosome will result in the microtubules of the barrel structure of each centriole to cut the electromagnetic field generated by the other centriole when rotating (Figure 1). Such a natural design makes centrosome to function as a molecular dynamo, generating directional electron flow through the dipolar structure of each individual microtubule in the centrosome, transforming the energy from ATP to electric current. During mitosis, centrosome is known to locate at the microtubule organizing center (MTOC), and only the mother centriole contains the sub-distal appendages that connect with spindle body microtubules, which allow the electrons to move from centrosome to the spindle body microtubules.

During mitosis chromosomes are connected with spindle body microtubules with K-fibres, which are microtubule bundles that join kinetochores to the spindle poles. Pericentriolar material (PCM) is composed primarily of hyaluronic acid (HA) and has a similar negative charge density as DNA [16]. The electrons flow over the spindle body leads to the electric excitation of the spindle body and chromosome, generating an enhanced intracellular electromagnetic field during mitosis, which is consistent with the observation of yeast cell at $\mathrm{M}$ phase [17] [18]. Bandyopadhyay et al. reported nano-sized electromagnetic pumping is required for microtubule self-assembly [13], the electric excitation of spindle body microtubules is required for the self-assembly and growth of the spindle body microtubules during $\mathrm{M}$ phase. The basal body of cilium is a centrosome like cellular organelle [19], similar molecular mechanism is applied for the basal body centriole to generate electric flow over the cilium microtubules, forming the nano electromagnetic field that is required for the self-assembly of cilium microtubule. Collectively, my hypothesis proposes centrosome is at the center of the electric network that continuously drawn the cellular chemical energy from ATP to feed the intracellular electromagnetic field. 


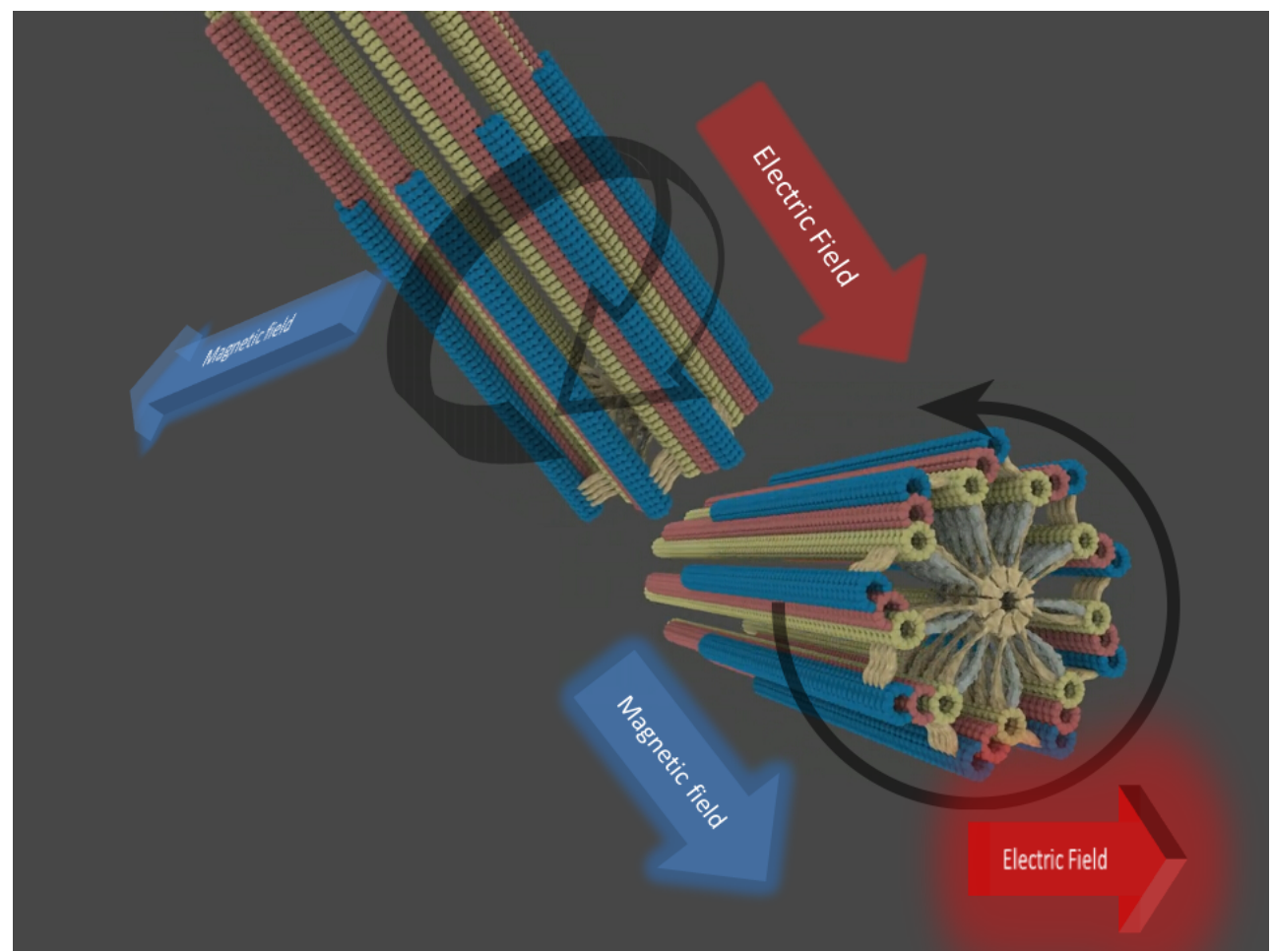

Figure 1. Cartoon illustration of how centrosome functions as a molecular dynamo within the living cell.

\section{Testing of the Hypothesis}

I am currently searching collaborators to do some experiment with the super resolution microscopy live cell imaging technique, hopefully to observe the rotation of barrel structure of the centrosome during mitosis in the living cell. Despite of current technical limitations, we still need to develop new methods for both direct measurements or experimental simulations of the intracellular electric field with advanced photonic and nano technologies.

This hypothesis is at the intersection of structural biology and quantum biology, which highlights a new avenue for basic biology researches. The hypothesis is supported by multiple lines of evidences and clearly elucidates the function-structure relationship of centrosome during mitosis for the first time, not only advance our knowledge to the function of centrosome but also have great implications for mitosis, one of the fundamental cellular events in biology.

\section{Conflict of Interests}

The author declares no conflict of interests for this hypothesis.

\section{Acknowledgements}

I thank Dr. Anirban Bandyopadhyay for his discussion with me about the hypothesis, and Dr. Laurent Schwartz for his interesting questions.

\section{References}

[1] Doxsey, S. (2001) Re-Evaluating Centrosome Function. Nature Review Molecular Cellular Biology, 2, 688-698. http://dx.doi.org/10.1038/35089575

[2] Bornens, M. and Azimzadeh, J. (2007) Origin and Evolution of the Centrosome. Advances in Experimental Medicine and Biology, 607, 119-129. http://dx.doi.org/10.1007/978-0-387-74021-8 10

[3] Bettencourt-Dias, M. and Glover, D. (2007) Centrosome Biogenesis and Function: Centrosomics Brings New Understanding. Nature Review Molecular Cellular Biology, 8, 451-463. http://dx.doi.org/10.1038/nrm2180 
[4] Mogensen, M.M. (1999) Microtubule Release and Capture in Epithelial Cells. Biology of the Cell, 91, 331-341. http://dx.doi.org/10.1111/j.1768-322X.1999.tb01091.x

[5] Chretien, D., Buendia, B., Fuller, S.D. and Karsenti, E. (1997) Reconstruction of the Centrosome Cycle from Cryoelectron Micrographs. Journal of Structural Biology, 120, 117-133. http://dx.doi.org/10.1006/jsbi.1997.3928

[6] Lawo, S., Hasegan, M., Gupta, G.D. and Pelletier, L. (2012) Subdiffraction Imaging of Centrosomes Reveals Higher-Order Organizational Features of Pericentriolar Material. Nature Cell Biology, 14, 1148-1158. http://dx.doi.org/10.1038/ncb2591

[7] Paintrand, M., Moudjou, M., Delacroix, H. and Bornens, M.J. (1992) Centrosome Organization and Centriole Architecture: Their Sensitivity to Divalent Cations. Journal of Structural Biology, 108, 107-128. http://dx.doi.org/10.1016/1047-8477(92)90011-X

[8] Bornens, M. (2002) Centrosome Composition and Microtubule Anchoring Mechanisms. Current Opinion in Cell Biology, 14, 25-34. http://dx.doi.org/10.1016/S0955-0674(01)00290-3

[9] Zhao, Y. and Zhan, Q. (2012) Electric Fields Generated by Synchronized Oscillations of Microtubules, Centrosomes and Chromosomes Regulate the Dynamics of Mitosis and Meiosis. Theoretical Biology and Medical Modelling, 9, 26. http://dx.doi.org/10.1186/1742-4682-9-26

[10] Zhao, Y. and Zhan, Q. (2012) Electric Oscillation and Coupling of Chromatin Regulate Chromosome Packaging and Transcription In Eukaryotic Cells. Theoretical Biology and Medical Modelling, 9, 27. http://dx.doi.org/10.1186/1742-4682-9-27

[11] Havelka, D., Kučera O., Deriu M.A. and Cifra M. (2014) Electro-Acoustic Behavior of the Mitotic Spindle: A Semi-Classical Coarse-Grained Model. PLoS ONE, 9, Article ID: e86501. http://dx.doi.org/10.1371/journal.pone.0086501

[12] Havelka, D., Cifra, M. and Kučera, O. (2014) Multi-Mode Electro-Mechanical Vibrations of a Microtubule: In Silico Demonstration of Electric Pulse Moving along a Microtubule. Applied Physics Letters, 104, Article ID: 243702. http://dx.doi.org/10.1063/1.4884118

[13] Sahu, S., Ghosh, S., Fujita, D. and Bandyopadhyay, A. (2014) Live Visualizations of Single Isolated Tubulin Protein Self-Assembly via Tunneling Current: Effect of Electromagnetic Pumping during Spontaneous Growth of Microtubule. Scientific Reports, 4, 7303. http://dx.doi.org/10.1038/srep07303

[14] Zimmerman, J.W., Pennison, M.J., Brezovich, I., et al. (2012) Cancer Cell Proliferation Is Inhibited by Specific Modulation Frequencies. British Journal of Cancer, 106, 307-313. http://dx.doi.org/10.1038/bjc.2011.523

[15] Hutson, R.L. (2015) Using the Electromagnetics of Cancer's Centrosome Clusters to Attract Therapeutic Nanoparticles. Advances in Bioscience and Biotechnology, 6, 172-181. http://dx.doi.org/10.4236/abb.2015.63017

[16] Van Oosten, A.S. and Janmey, P.A. (2013) Extremely Charged and Incredibly Soft: Physical Characterization of the Pericellular Matrix. Biophysical Journal, 104, 961-963. http://dx.doi.org/10.1016/j.bpj.2013.01.035

[17] Jelínek, F., Cifra, M., Pokorný, J., et al. (2009) Measurement of Electrical Oscillations and Mechanical Vibrations of Yeast Cells Membrane around 1 kHz. Electromagnetic Biology and Medicine, 28, 223-232. http://dx.doi.org/10.1080/15368370802710807

[18] Pohl, H.A., Braden, T., Robinson, S., et al. (1981) Life Cycle Alternations of the Micro-Dielectrophoretic Effects in Cells. Journal of Biological Physics, 9, 133-154. http://dx.doi.org/10.1007/BF01988247

[19] Adams, M. (2010) The Primary Cilium: An Orphan Organelle Finds a Home. Nature Education, 3, 54.

\section{Abbreviations}

EMF electromagnetic field

MTOC microtubule organizing center

PCM Pericentriolar material 